\title{
Potential protective effect of ALDH-1 stromal expression against early recurrence of operable breast cancers
}

\author{
Jung Sun Lee ${ }^{1}$, Woo Gyeong Kim ${ }^{2}$ \\ ${ }^{1}$ Department of Surgery, ${ }^{2}$ Department of Pathology, Haeundae Paik Hospital, College of Medicine, Inje University, Busan 48108, Korea \\ Contributions: (I) Conception and design: JS Lee; (II) Administrative support: None; (III) Provision of study materials or patients: None; (IV) \\ Collection and assembly of data: JS Lee; (V) Data analysis and interpretation: All authors; (VI) Manuscript writing: All authors; (VII) Final approval \\ of manuscript: All authors. \\ Correspondence to: JungSun Lee, MD, PhD. Department of Surgery, Haeundae Paik Hospital, College of Medicine, Inje University, 1435 Jwa-dong, \\ Haeundae-gu, Busan 48108, Korea. Email: gsjslee@gmail.com.
}

\begin{abstract}
Background: The emerging cancer stem cell (CSC) model proposes that the stem cell niche plays a major role in the risk of cancer recurrence. Enzymatic activity of aldehydes, including aldehyde dehydrogenase 1 (ALDH-1), has been used as a marker of normal and malignant breast stem cells (BSCs). However, the clinical implications of the expression of stem cell markers in the stroma have not yet been investigated.

Methods: To determine the relationships of ALDH-1 expression, the currently reliable BSCs marker, with clinical characteristics and survival, we used immunohistochemical staining of tissue microarrays from 180 stroma and epithelial cancer tissues in patients diagnosed with operable early breast cancer (stage 0-III).

Results: ALDH-1 expression was observed in $93.4 \%$ of the stromal cells and in $37.2 \%$ of the epithelial cells, and the expression levels between the two cell types were significantly correlated $(\mathrm{P}=0.001)$. The stromal expression of ALDH-1 was not correlated with any clinical factors, whereas epithelial expression was significantly correlated with a negative estrogen-receptor status $(\mathrm{P}<0.001)$, high proliferation based on Ki-67 expression $(\mathrm{P}<0.001)$, and younger age $(\mathrm{P}=0.04)$. After 27.8 months of follow up, negative stromal expression of ALDH-1 was significantly correlated with shorter overall survival (positive, $56.9 \pm 3.0$ months vs. negative, $30.5 \pm 3.0$ months; $\mathrm{P}=0.01$ ).

Conclusions: Stromal ALDH-1 expression was not directly correlated with known clinical factors, but its expression may play a protective role against early recurrence. Further observation and large-scale studies are needed to validate the clinical implications of ALDH-1 in breast cancer.
\end{abstract}

Keywords: Breast neoplasm; prognosis; stem cell niche

Received: 01 September 2018; Accepted: 29 September 2018; Published: 29 November 2018.

doi: $10.21037 /$ sci.2018.11.03

View this article at: http://dx.doi.org/10.21037/sci.2018.11.03

\section{Introduction}

Cancer stem cells (CSCs) may serve as the unit of selection in the genetic evolution of tumors; however, CSCs are known to be genetically unstable, generating multiple clones consisting of genetically altered cells and their differentiated progeny. Cancer cells display a remarkable degree of phenotypic plasticity, and there is evidence that more differentiated tumor cells may acquire stem cell properties through a dedifferentiation process, which has significant clinical implications (1).
It is now widely accepted that the tumor microenvironment is a pathologically active niche that shapes the tumor's nature, evolution, and response to treatment. Close interactions between cancer cells and the stroma are known to regulate several cancer pathways; thus, the determination of different tumorstromal interactions could be an important step in invasiveness. The breast cancer microenvironment is a complex combination of several different cell types and molecules, and is a key contributor to tumor development and progression. The microenvironment includes 
fibroblasts, macrophages, immune cells, tumor-infiltrating lymphocytes, endothelial cells, and angiogenic vascular cells, whereas stroma cells surround and directly interact with tumor cells.

Recent work has shone new light on the role of the niche, such as stromal cells, for epithelial organ maintenance and regeneration through the simultaneous production of proliferative and differentiative cues. Thus, previous CSC model which means cancer progression involves gradual loss of a differentiated phenotype and acquisition of stemcell-like features should perhaps be expanded to include the possibility that niche cells also contribute factors required for CSC differentiation, progression and treatment failure (2). Indeed, several studies have identified a clear role for the stroma, which includes the niche cells for several populations of epithelial stem cells, in the restraint of epithelial cancer growth and progression in multiple organs, including the bladder, pancreas, colon, and prostate (3-5). Since cancer cells already have proliferation-promoting genetic lesions, thereby obviating the need for stromally secreted proliferative signals, the stromal niche-derived differentiation signals are less well defined but may play a critical role in restraining cancer growth and progression (3-5).

Aldehyde dehydrogenase 1 (ALDH-1) is a major enzyme involved in the synthesis of retinoic acid (7), which shows antiproliferative activity on breast cancer cells $(6,7)$. The expression of ALDH-1 in the breast is not restricted to the epithelial cells, and has also been noted in stromal fibroblasts $(8-11)$. Resetkova et al. $(11,12)$ analyzed the stromal ALDH-1 expression in two independent triplenegative cohorts, and found that high stromal ALDH-1 expression was significantly associated with better diseasefree survival (DFS) with a trend for longer overall survival (OS) with a median of 67 months. Using different markers, Farmer et al. (13) showed that in patients with breast cancer who received adjuvant chemotherapy, higher expression of the stromal metagene (ESR1, CLCA2, DCN, GZMA, CD83, FABP4, TPX2, $M X 1$, and $A D M$ ) was associated with significantly shorter relapse-free survival times. These studies thus support the hypothesis of a strong influence of the stromal content on resistance to chemotherapy. Similarly, Louhichi et al. (14) found that CD10 expression by stromal cells, but not neoplastic cells, correlated significantly with the expression of the CSC markers CD44+/ALDH-1+, supporting a role of stromal CD10 expression in breast cancer progression and dissemination, and suggesting a relationship with CSCs.

ALDH-1 has a functional role in stem cell differentiation, and measurement of ALDH-1 enzymatic activity has been shown to distinguish benign and malignant stem cells in breast cancer and other neoplasms, including human hematopoietic malignancies. Moreover, expression of ALDH-1 in the cytoplasm of cancer cells is associated with a poor clinical outcome and increased breast cancer risk in normal breast tissues. Kunju et al. (9) reported that ALDH-1 was expressed in both epithelial and stromal cells in benign breast tissues, and that ALDH-1 positivity in both cell types is associated with an increased risk of breast cancer. In contrast, Isofoss et al. (15) demonstrated that the absence of ALDH-1-positive cells in the benign mammary stroma was associated with increased risk factors for breast cancer. However, the clinical significance of ALDH-1 in breast cancer has rarely been evaluated.

In this study, we analyzed ALDH-1 expression in both the epithelial cells and stromal cells of tissue specimens obtained from patients with operable early breast cancer to support the clinical evidence of stromal ALDH-1 expression in playing a protective role against early breast cancer recurrence.

\section{Methods}

\section{Patients and data collection}

The collection of breast cancer patient data and tissues was performed according to the guidelines of the Inje biobank. All participating patients signed informed consent forms approved by the responsive authority before removal of primary tumors. Data from 188 patients who underwent surgery for breast cancer between October 2013 and February 2014 at Haeundae paik Hospital were retrospectively reviewed. Patients who underwent neoadjuvant chemotherapy, diagnosed with ipsilateral breast tumor recurrence, or underwent excisional biopsy at another institute were excluded from the study. After the first exclusion, a total of 180 cases were included for analysis. We then further excluded patients who were undertreated owing to older age, side effects of adjuvant treatment, and personal phobia of adjuvant treatment, resulting in a total of 168 cases included for assessment of survival outcomes. This study was approved by the Institutional Review Board of Haeundae-Paik Hospital, Inje University (IRB number: Haeundae-paik 2013-60) and was conducted in accordance with the Declaration of Helsinki. 

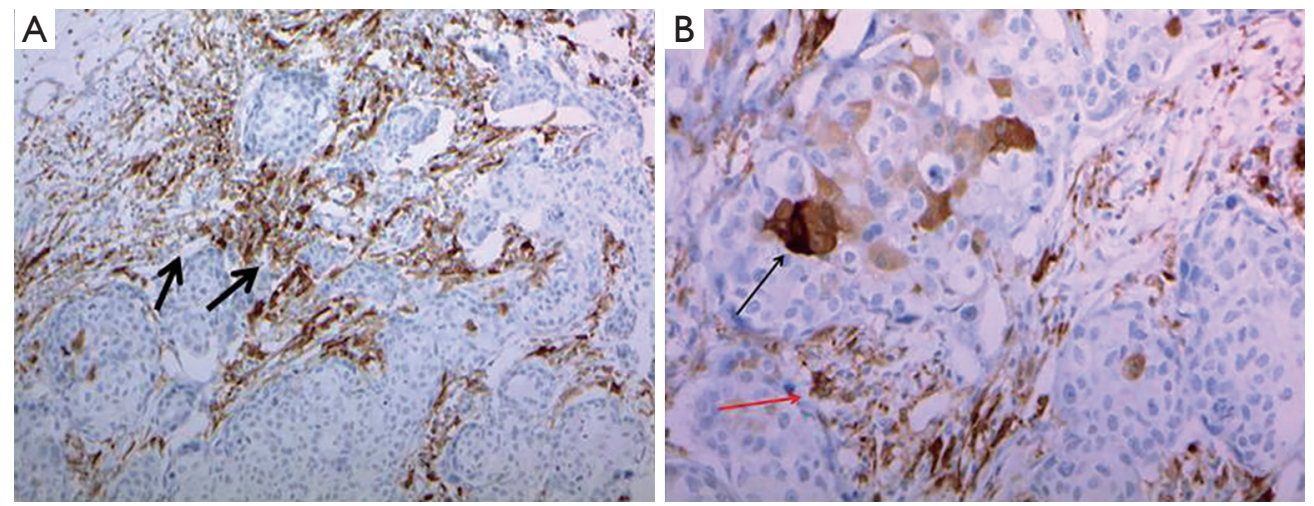

Figure 1 Immunohistochemical staining of ALDH-1 in breast cancer tumor sections. (A) This section shows ALDH-1 expression in peritumoral stromal cells in invasive ductal carcinoma (black arrows, magnification $\times 200$ ); (B) this section shows ALDH-1 expressions in both tumor epithelial cells (red arrow) and stromal cells (black arrow) in the same breast cancer specimen (magnification $\times 400$ ).

\section{Immunohistochemistry of ALDH-1 in stromal and epithelial cells}

ALDH-1 antibody (1:100 dilution; clone 44/ALDH, BD Biosciences, San Jose, CA, USA) was used for ALDH-1 immunostaining. Staining was performed using the Ventana Ultraview Universal DAB kit (Ventana Medical System, Inc, Tucson, AZ, USA) according to the manufacturer's protocols. Cytoplasmic staining of cancer cells was considered to reflect an ALDH-1 (+) status. A positive control for the specificity of ALDH-1 staining was carried out with paraffin sections of normal hepatic tissue, which shows diffuse strong cytoplasmic expression. To evaluate the relevance of ALDH-1 expression in epithelial tumor and stromal cells, these components were evaluated in both whole tumor sections and in large core $(1 \mathrm{~mm})$ tissue microarrays of all primary breast cancers (Figure 1).

\section{Immunobistochemistry scoring}

All cases were independently reviewed and scored by a single pathologist (WGK) who was blinded to the clinical diagnosis. To compare the present results with previous studies reporting a large series of invasive ductal carcinoma cases, expression levels of ALDH-1 in both the stroma and epithelium of the breast were evaluated and scored as previously described $(16,17)$. Staining of ALDH-1 was classified as $4+(75-100 \%$ positive tumor cells), $3+(75-50 \%), 2+(10-50 \%), 1+(1-10 \%)$, and $0(0 \%)$ in both epithelial tumor cells and stromal cells. For subsequent analyses, any epithelial tumor cells with positive staining was considered to have ALDH-1 (+) expression $(16,18)$.

\section{Statistical analysis}

Statistical analyses were performed to assess the relationships between clinical variables and the expression of ALDH-1 in both epithelial tumor and stromal cells. Differences between categorical variables were evaluated using standard chi-squared tests or McNemar tests. The log-rank (Mantel-Cox) test was used to determine the effect of stromal or epithelial expression of ALDH-1 on DFS (recurrence or death due to breast cancer) and OS (death from any cause). Values are expressed as the mean \pm standard deviation. All analyses were performed using SAS (version 9.4, SAS, Inc., Cary, NC), and P values less than 0.05 were considered to be statistically significant.

\section{Results}

\section{ALDH-1 expression is more frequent in epithelial tumor cells and correlated with stromal cell expression}

Positive stromal ALDH-1 expression was detected in 94.4\% (169/179) of the 180 patients, whereas epithelial ALDH-1 expression was only detected in $38.2 \%$ of these patients (Table 1). Epithelial ALDH-1 expression was significantly associated with a negative estrogen receptor status, younger patients, high Ki-67 expression, and specific breast cancer subtype $(\mathrm{P}=0.001$; Tables 2 and 3$)$. No correlation between stromal expression of ALDH-1 and any of the clinicopathologic factors was detected (Table 2). Moreover, epithelial ALDH-1 expression of breast cancer was also correlated with stromal ALDH-1 expression $(\mathrm{P}<0.001 ;$ Table 4). 
Table 1 Clinicopathological features of 180 breast carcinomas

\begin{tabular}{|c|c|c|}
\hline Characteristics & Categories & $\begin{array}{l}\text { Number of } \\
\text { patients (\%) }\end{array}$ \\
\hline \multirow[t]{3}{*}{ Size of tumors $(\mathrm{cm})$} & Mean $(\mathrm{cm})$ & 2.27 \\
\hline & SD & 1.34 \\
\hline & Range (cm) & $0.2-8.0$ \\
\hline \multirow[t]{5}{*}{ Histologic type of tumors } & Ductal, in situ & $7(3.9)$ \\
\hline & Ductal, invasive & $162(90.0)$ \\
\hline & Lobular, invasive & $4(2.2)$ \\
\hline & Lobular, in situ & $1(0.6)$ \\
\hline & Etc. & $6(3.3)$ \\
\hline \multirow[t]{3}{*}{ Nottingham grade } & Grade 1 & $22(12.2)$ \\
\hline & Grade 2 & $70(38.9)$ \\
\hline & Grade 3 & $87(48.3)$ \\
\hline \multicolumn{3}{|l|}{ Hormone receptors } \\
\hline \multirow[t]{2}{*}{ ER } & Negative & $91(50.6)$ \\
\hline & Positive & $89(49.4)$ \\
\hline \multirow[t]{2}{*}{ PR } & Negative & $120(66.7)$ \\
\hline & Positive & 60 (33.3) \\
\hline \multirow[t]{2}{*}{ Her-2/neu } & Not overexpressed & $131(72.8)$ \\
\hline & Overexpressed & $49(27.2)$ \\
\hline \multirow[t]{4}{*}{ Stage } & 0 & $7(3.9)$ \\
\hline & 1 & $91(50.6)$ \\
\hline & II & $75(41.7)$ \\
\hline & III & $7(3.9)$ \\
\hline \multirow[t]{3}{*}{ Lymph node status } & Negative & $115(63.9)$ \\
\hline & Positive & $52(28.9)$ \\
\hline & Not assessed & $13(7.2)$ \\
\hline \multirow[t]{2}{*}{ Stromal ALDH-1 } & Negative & $10(5.6)$ \\
\hline & Positive & $169(94.4)$ \\
\hline \multirow[t]{2}{*}{ Epithelial ALDH-1 } & Negative & $112(61.8)$ \\
\hline & Positive & $68(38.2)$ \\
\hline
\end{tabular}

$\mathrm{SD}$, standard deviation; ER, estrogen receptor; PR, progesterone receptor.

\section{Negative stromal ALDH-1 expression was correlated with early recurrence of breast cancer}

The median duration of follow up in the 168 patients included for survival assessment was $27.8 \pm 10.2$ months.
The log-rank (Mantel-Cox) test showed that positive stromal ALDH-1 expression was correlated with longer OS (positive, 56.9 \pm 3.0 months $v s$. negative, $30.5 \pm 3.0$ months; $\mathrm{P}=0.01)$, whereas epithelial ALDH-1 expression was not correlated with survival $(\mathrm{P}=0.36)$ (Table 5) (Figure 2). However, not only stromal ALDH-1 expression (positive, $60.3 \pm 2.65$ months vs. negative, $30.4 \pm 2.46$ months; $\mathrm{P}=0.10$ ) but also epithelial ALDH-1 expression (positive, $52.4 \pm 0.6$ months $v$ s. negative, $58.9 \pm 3.8$ months; $\mathrm{P}=0.30$ ) was not correlated with disease free survival (Table 6) (Figure 2).

\section{Discussion}

Over the past few years, experiments involving lineage tracing and cell ablation in intact tumors have confirmed that many of these tumors harbor stem cells in dedicated niches (19-23). In the case of the mammary gland, Liu et al. $(24,25)$ showed that breast cancer stem cells (BCSCs) exist in a distinct mesenchymal-like (epithelialmesenchymal transition, EMT) and epithelial-like (mesenchymal-epithelial transition) state. Mesenchymallike BCSCs, characterized by CD44+CD24- expression, are primarily quiescent and localized at the tumor invasive front, whereas epithelial-like BCSCs that express ALDH are proliferative, and are located more centrally. Using immunohistochemistry analysis, Currie et al. (23) showed that CD44+/CD24- and CD133+ tumor cells were more common in areas of hypoxia, whereas ALDH-1+ tumor cells were more frequently associated with regions of high tumor microvascular density. This study supported the concept that the tumor microenvironment determines the phenotypic plasticity of breast cancer stem-like cells and has important clinical implications with respect to the development of therapeutic strategies aimed at cancer cells with stem-like properties. Expansion of the stromal cell population expressing ALDH-1 beyond the intralobular stroma has also been associated with cancer development $(8,12,26)$. Taken together, these data suggest a role for the microenvironment in promoting tumor development before morphological abnormalities of carcinoma become evident. Moreover, these findings support the utility of immunohistochemistry in the evaluation of ALDH-1 as a putative marker of BCSCs. In addition, given that stromal gene expression appears to be broadly altered in breast cancer, it will be interesting to further examine whether alterations in stromal niche signaling programs may play a role in breast cancer development and progression $(27,28)$.

Stem cell markers reflect adverse biomarker profiles 
Table 2 Correlations between expression of breast ALDH-1in both epithelium and stroma according to clinicopathologic features

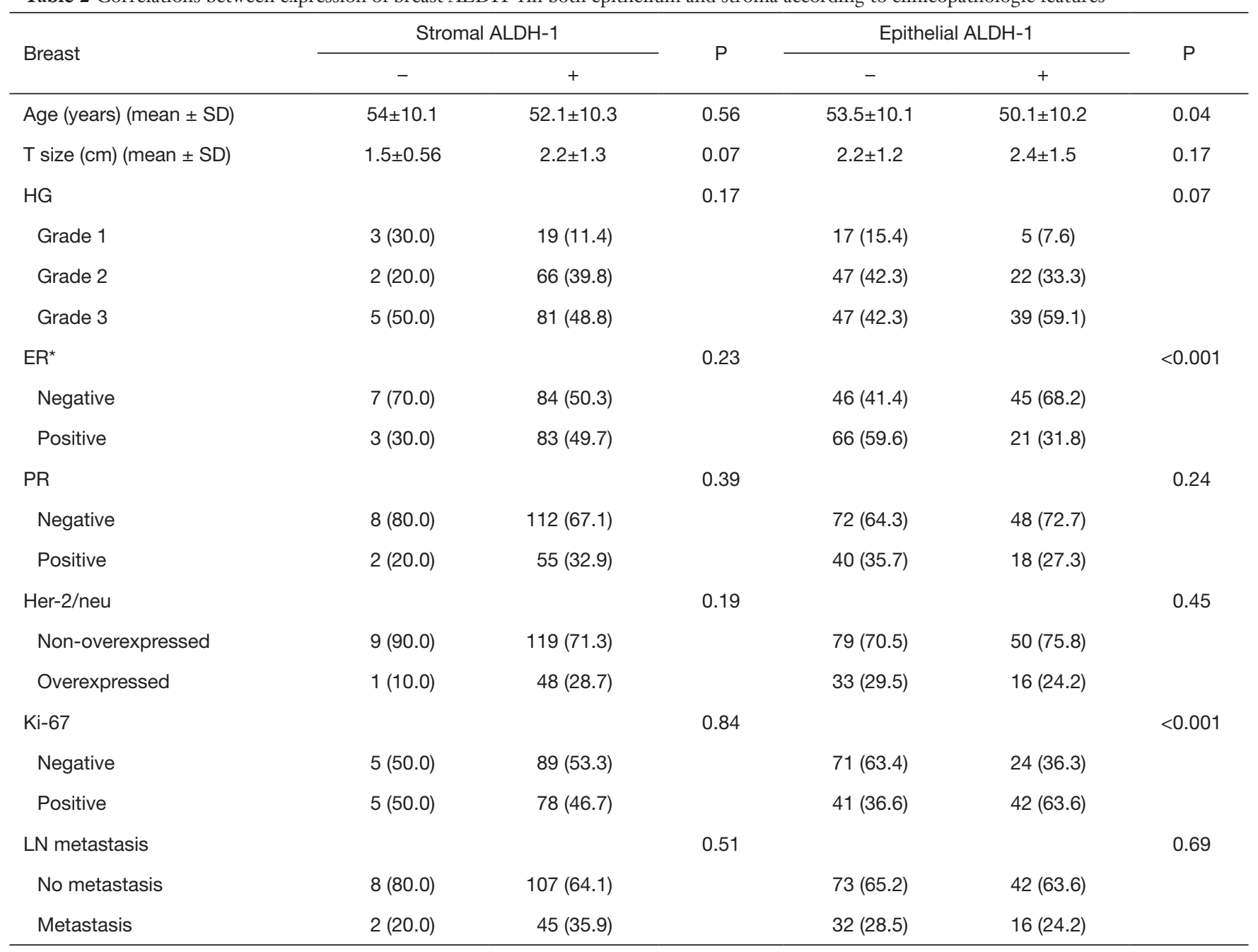

*, McNemar test. SD, standard deviation; HG, histologic grade; ER, estrogen receptor, PR, progesterone receptor; LN, lymph node.

Table 3 Correlations between expression of ALDH-1 of both epithelium and stroma according to breast cancer subtype

\begin{tabular}{|c|c|c|c|c|c|c|c|}
\hline Breast & Luminal A & Luminal B (HER2-) & Luminal B (HER2+) & HER2+ & Basal-like & Total & $\mathrm{P}$ \\
\hline- & $1(10.0)$ & $1(10.0)$ & $1(10.0)$ & $1(10.0)$ & $6(60.0)$ & 10 & \\
\hline+ & $39(22.8)$ & $22(12.6)$ & $31(18.6)$ & $36(21.6)$ & $41(24.6)$ & 167 & \\
\hline Epithelial ALDH-1 & & & & & & & 0.003 \\
\hline+ & $11(16.7)$ & $8(12.1)$ & $5(7.6)$ & $14(21.2)$ & $28(42.4)$ & 66 & \\
\hline
\end{tabular}

and molecular subtypes of breast cancer (29). In addition, the stem cell markers CD24, CD44, ALDH-1, and SOX2 could identify different cancer cell sub-populations with no consistent therapeutic implication, rather than a single population of cells by commonly employed methods (29). With this method, the relationships of BCSCs with clinical 
Table 4 Correlations stromal expression of ALDH-1 and epithelial expression of ALDH-1

\begin{tabular}{lcccc}
\hline \multirow{2}{*}{ CSC marker } & \multicolumn{2}{c}{ Epithelium } & \multirow{2}{*}{ Total } & $\mathrm{P}^{\S}$ \\
\cline { 2 - 3 } & ALDH-1 (-) & ALDH-1 (+) & & \\
\hline Stroma & & & & $<0.001$ \\
ALDH-1 (-) & $9(8.1)$ & $1(1.5)$ & 10 & \\
ALDH-1 (+) & $102(91.9)$ & $65(98.5)$ & 167 & \\
Total & 111 & 66 & 177 & \\
\hline
\end{tabular}

§, McNemar test. CSC, cancer stem cell;
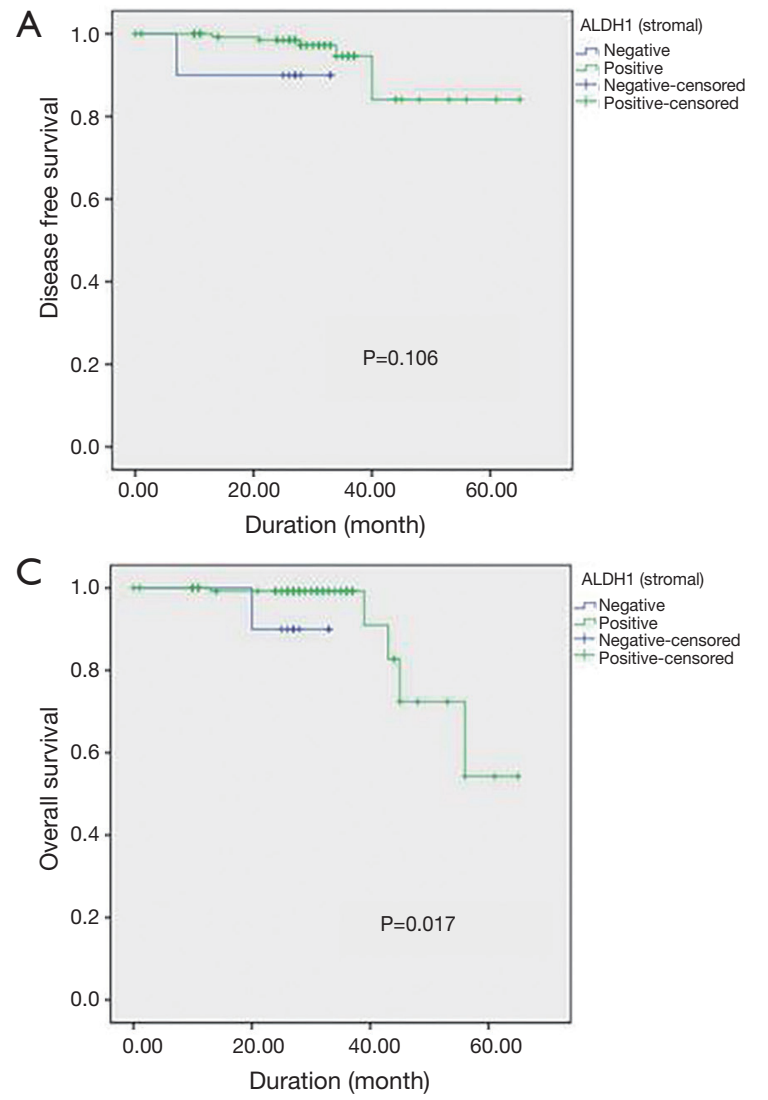

Table 5 Mean overall survival according to expression of CSC marker between stromal and epithelial tissue

\begin{tabular}{lcccc}
\hline CSC marker & $\begin{array}{c}\text { Mean } \\
\text { survival time }\end{array}$ & SD & $95 \% \mathrm{Cl}$ & $\mathrm{P}$ \\
\hline Stromal ALDH-1 & & & & 0.01 \\
$\quad$ Negative & 30.5 & 2.3 & $25.8,35.1$ & \\
$\quad$ Positive & 56.9 & 3.0 & $50.9,62.9$ & \\
$\quad$ Total & 56.6 & 3.0 & $50.6,62.7$ & \\
Epithelial ALDH-1 & & & & 0.36 \\
Negative & 55.1 & 4.0 & $47.0,63.0$ & \\
Positive & 56.0 & 0 & $56.0,56.0$ & \\
$\quad$ Total & 56.7 & 3.1 & $50.6,62.7$ & \\
\hline
\end{tabular}
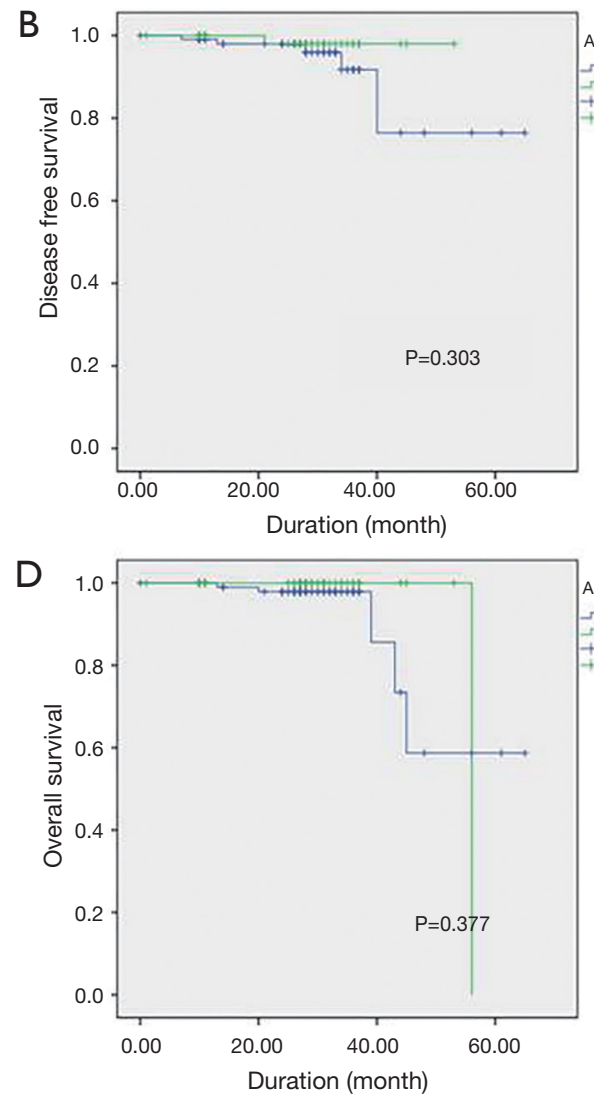

Figure 2 Disease-free survival (DFS) according to (A) stromal ALDH-1 expression and (B) epithelial ALDH-1 expression. Overall survival (OS) according to (C) stromal ALDH-1 expression and (D) epithelial ALDH-1 expression. Blue lines show patients with negative ALDH-1 expression and green lines show patients with positive ALDH-1 expression. 


\begin{tabular}{|c|c|c|c|c|}
\hline CSC marker & $\begin{array}{c}\text { Mean } \\
\text { survival time }\end{array}$ & $\mathrm{SD}$ & $95 \% \mathrm{Cl}$ & $P$ \\
\hline Stromal ALDH-1 & & & & 0.10 \\
\hline Negative & 30.4 & 2.46 & $25.6,35.2$ & \\
\hline Positive & 60.3 & 2.65 & $55.2,65.6$ & \\
\hline Total & 60.1 & 2.65 & $54.8,65.3$ & \\
\hline Epithelial ALDH-1 & & & & 0.30 \\
\hline Negative & 58.0 & 3.8 & $50.6,65.4$ & \\
\hline Positive & 52.4 & 0.6 & $51.1,53.6$ & \\
\hline Total & 60.1 & 2.6 & $54.9,65.2$ & \\
\hline
\end{tabular}

parameters will require identification of specific markers or panels for the individual cancer type. However, there is a limitation of this approach with regards to cancer plasticity and heterogeneity. Moreover, the results of context-based studies and new study models should be considered to best illustrate a corresponsive principle on the relationship between stroma and epithelial tumor cells in bulk tumors. Malta et al. (30) suggested adopting a connectivity map, which is a data-driven, systematic approach for discovering associations among genes, chemicals, and biological conditions associated with stemness in more than 10 cancer types. However, they did not consider the property of stromal stemness in these cancer types.

In conclusion, although we found that epithelial ALDH-1 expression was associated with poor prognostic factors, it was not directly correlated with early clinical outcomes. This study has several limitations, including the short observation period and small sample size. It is possible that longer-term observation would result in similar OS rates between the groups with positive and negative epithelial tumor cell ALDH-1 expression. Despite these limitations, our findings suggest that stromal ALDH-1 breast cancers could be correlated with a good prognosis and longer OS. After standard adjuvant treatment, stromal stemness may act as a brake against early treatment failure; however, more in-depth investigation is needed to confirm the value of ALDH-1 as a candidate prognostic marker. In addition, stromal cells are composed of several cell types, including mast cells, fibroblasts, and immune cells, and thus further studies are needed to develop methods for identifying the specific stromal cell types.

\section{Acknowledgements}

The biospecimens and data used in this study were provided by the Biobank of InJe University PAIK Hospital (InJeBiobank), a member of Korea Biobank Network. Also, this work was supported by a grant from Research year of Inje University in 2017.

Funding: The present study was funded by the Korean Breast Cancer Foundation (KBCF-2013E006).

\section{Footnote}

Conflicts of Interest: The authors have no conflicts of interest to declare.

Ethical Statement: This study was approved by the Institutional Review Board of Haeundae-Paik Hospital, Inje University (IRB number: Haeundae-paik 2013-60) and was conducted in accordance with the Declaration of Helsinki.

\section{References}

1. Brooks MD, Burness ML, Wicha MS. Therapeutic implications of cellular heterogeneity and plasticity in breast cancer. Cell Stem Cell 2015;17:260-71.

2. Shin K, Lim A, Zhao C, et al. Hedgehog signaling restrains bladder cancer progression by eliciting stromal production of urothelial differentiation factors. Cancer Cell 2014;26:521-33.

3. Gerling M, Büller NV, Kirn LM, et al. Stromal Hedgehog signalling is downregulated in colon cancer and its restoration restrains tumor growth. Nat Commun 2016;7:12321

4. Lee JJ, Perera RM, Wang H, et al. Stromal response to Hedgehog signaling restrains pancreatic cancer progression. Proc Natl Acad Sci USA 2014;111: E3091-100.

5. Lim A, Shin K, Zhao C, et al. Spatially restricted Hedgehog signalling regulates HGF-induced branching of the adult prostate. Nat Cell Biol 2014;16:1135-45.

6. Yang Q, Sakurai T, Kakudo K. Retinoid, retinoic acid receptor beta and breast cancer. Breast Cancer Res Treat 2002;76:167-73.

7. Yoshida A, Hsu LC, Dave V. Retinal oxidation activity and biological role of human cytosolic aldehyde dehydrogenase. Enzyme 1992;46:239-44.

8. Ginestier C, Hur MH, Charafe-Jauffret E, et al. ALDH1 is a marker of normal and malignant human mammary 
stem cells and a predictor of poor clinical outcome. Cell Stem Cell 2007;1:555-67.

9. Kunju LP, Toy KA, Thomas DG, et al. Expression of the stem cell markers ALDH1 and EZH2 in triple negative invasive breast carcinomas. [Abstracts of the annual meeting of the united states and canadian academy of pathology. March 7-13, 2009. Boston, Massachusetts, USA]. Lab Invest 2009;89:52A.

10. Lim E, Vaillant F, Wu D, et al. Aberrant luminal progenitors as the candidate target population for basal tumor development in BRCA1 mutation carriers. Nat Med 2009;15:907-13.

11. Resetkova E, Thorat MA, Badve S. Expression of ALDH1 (putative stem cell marker) does not correlate with response to neo-adjuvant chemotherapy (NACT) in breast carcinoma. [Abstracts of the XXVIIth international congress of the international academy of pathology. October 12-17, 2008. Athens, Greece]. Histopathology 2008;53:159.

12. Resetkova E, Reis-Filho JS, Jain RK, et al. Prognostic impact of ALDH1 in breast cancer: a story of stem cells and tumor microenvironment. Breast Cancer Res Treat 2010;123:97-108.

13. Farmer P, Bonnefoi H, Anderle P, et al. A stromarelated gene signature predicts resistance to neoadjuvant chemotherapy in breast cancer. Nat Med 2009;15:68-74.

14. Louhichi T, Saad H, Dhiab MB, et al. Stromal CD10 expression in breast cancer correlates with tumor invasion and cancer stem cell phenotype. BMC Cancer 2018;18:49.

15. Isfoss BL, Holmqvist B, Sand E, et al. Stellate cells and mesenchymal stem cells in benign mammary stroma are associated with risk factors for breast cancer- and observational study. BMC Cancer 2018;18:230.

16. de Beça FF, Caetano P, Gerhard R, et al. Cancer stem cells markers CD44, CD24, and ALDH-1 in breast cancer special histological types. J Clin Pathol 2013;66:187-91.

17. Ricardo S, Vieira AF, Gerhard R, et al. Breast cancer stem cell markers CD44, CD24 and ALDH-1: expression distribution within intrinsic molecular subtype. J Clin Pathol 2011;64:937-46.

18. Deng S, Yang X, Lassus H, et al. Distinct expression levels and patterns of stem cell marker, aldehyde dehydrogenase isoform 1 (ALDH1), in human epithelial cancers. PLoS One 2010;5:e10277.

19. Driessens G, Beck B, Caauwe A, et al. Defining the mode of tumour growth by clonal analysis. Nature 2012;488:527-30.

20. Schepers AG, Snippert HJ, Stange DE, et al. Lineage tracing reveals Lgr5 + stem cell activity in mouse intestinal adenomas. Science 2012;337:730-5.

21. Kozar S, Morrissey E, Nicholson AM, et al. Continuous clonal labeling reveals small numbers of functional stem cells in intestinal crypts and adenomas. Cell Stem Cell 2013;13:626-33.

22. Chen J, Li Y, Yu TS, et al. A restricted cell population propagates glioblastoma growth after chemotherapy. Nature 2012;488:522-6.

23. Currie MJ, Beardsley BE, Harris GC, et al. Immunohistochemical analysis of cancer stem cell markers in invasive breast carcinoma and associated ductal carcinoma in situ: relationship with markers of tumor hypoxia and microvascularity. Hum Pathol 2013;44:402-11.

24. Liu S, Clouthier SG, Wicha MS. Role of microRNAs in the regulation of breast cancer stem cells. J Mammary Gland Biol Neoplasia 2012;17:15-21.

25. Gupta PB, Fillmore CM, Jiang G, et al. Stochastic state transitions give rise to phenotypic equilibrium in populations of cancer cells. Cell 2011;146:633-44.

26. Hess DA, Wirthlin L, Craft TP, et al. Selection based on CD133 and high aldehyde dehydrogenase activity isolates long-term reconstituting human hematopoietic stem cells. Blood 2006;107:2162-9.

27. Finak G, Bertos N, Pepin F, et al. Stromal gene expression predicts clinical outcome in breast cancer. Nat Med 2008;14:518-27.

28. Roberts KJ, Kershner AM, Beachy PA. The stromal niche for epithelial stem cells: a template for regeneration and a brake on malignancy. Cancer Cell 2017,32:404-10.

29. Liu Y, Nenutil R, Appleyard MV, et al. Lack of correlation of stem cell markers in breast cancer stem cells. $\mathrm{Br} \mathrm{J}$ Cancer 2014;110:2063-71.

30. Malta TM, Sokolov A, Gentles AJ, et al. Machine learning identified stemness features associated with oncogenic dedifferentiation. Cell 2018;173:338-54.e15. doi: $10.21037 /$ sci.2018.11.03

Cite this article as: Lee JS, Kim WG. Potential protective effect of ALDH-1 stromal expression against early recurrence of operable breast cancers. Stem Cell Investig 2018;5:43. 